\title{
An Advanced Pavement Management System based on a Genetic Algorithm for a Motorway Network
}

\author{
G. Di Mino ${ }^{1}$, M.R. De Blasiis ${ }^{2}$, F. Di Noto ${ }^{3}$ and S. Noto ${ }^{1}$ \\ ${ }^{1}$ University of Palermo, Italy \\ ${ }^{2}$ University of Roma3, Italy \\ ${ }^{3}$ SPEA Ingegneria Europea, Italy
}

\begin{abstract}
Maintenance and improvement, through the rehabilitation, of the road infrastructure is a strategic and priority objective for road agencies, nevertheless the economic resources required are often inadequate.

Within road management, the pavement management system (PMS) plays an essential role because of both the money needed and the performance that should be provided in terms of safety, ride quality and transport cost.

The PMS is based on searching for a balanced solution between the lowest cost and the increased level of performance (i.e. pavement condition).

In this paper a PMS multi-objective optimization method, was proposed, using a genetic algorithm (GA) to identify the best solution considering different rehabilitation strategies. The multi-objective optimization GA permits a set of optimal solutions (the Pareto solution set) that takes into account all the considered constraints.

Finally on the basis of a specific criteria the best solution was selected in relation to the ranking of the priorities of the agency.

A detailed numerical study was conducted on the Italian A18 motorway and the results showed that the proposed model PMS-GA is a suitable support to the decision making process.
\end{abstract}

Keywords: pavement management system, genetic algorithm, multi-objective optimization.

\section{Introduction}

All over the world the life cycle cost analysis (LCCA), born in the USA, is the most successful method to identify the best investment for road pavement maintenance and rehabilitation strategies. This method implies the knowledge of several parameters: the investment discount rate, the life design of the road pavement, the 
users and agency cost in order to select the best strategy under given budget constraint. However the implementation of the LCCA in other countries different from USA could be not feasible since the conditions as the way and the term of financing, the laws for public works, the agencies and the construction process are peculiar in every country. Therefore the researchers aim at developing advanced models in order to provide a suitable support to decision making process. As a result, there are many specific software sometimes implemented by the same agencies.

However government agencies and private ones have to accept the necessary budget for rehabilitation would often turn out much bigger than the available one.

By the light of the lack of economical resources generally the agencies of national motorways and highways have to carry out a optimization process regarding two opposite goals: major road safety and minor expenditure.

Therefore the decision making process has to be based on well-grounded criteria being able to select the best strategy aimed to reduce the number of the road accidents due to the improvement of pavement conditions, under the constraint of the annual budget for rehabilitation.

Conceptually speaking the above-mentioned issue can be tackled by a multiobjectives approach based on genetic algorithms (GAs) which is also a powerful tool to manage huge data in reasonable time.

The aim of the research was to develop a two-objectives optimization model, both on network level and project one, which considers road accident risk and rehabilitation costs as objective functions to be minimised. A GA based procedure has been developed in order to obtain the near optimal solution in terms both of cost and effectiveness.

The model was performed on real case concerning the A18 Messina-Catania motorway in Italy and the results demonstrated that the GAs procedure was able to reach technical and economical targets.

\section{Overview on Genetic Algorithm}

The GAs is substantially an optimization technique based on principle of natural selection. GAs operate in order to allow the evolution of a population (space of solutions), composed by C-individuals called chromosomes, towards one or more peculiar goals (objective functions). Every chromosome is encoded by $\mathrm{N}$-genes and is ranked by a F-fitness value that measures its effectiveness to reach the target. The initial population (parents) generates a new population (spring-off) by means of several operator, such as reproduction, roulette wheel selection, tournament selection, mutation, inversion, cross-over; this process keeps on running for Ggenerations. The evolution is allowed by the fact that some spring-off chromosomes have better F-fitness value than those of their parents. By the light of the heuristic nature of the process GAs don't guarantee to find the optimal solution of a given problem, but usually an "near optimal solution" can be obtained after a relatively few number of generations (quickly convergence of solution). The convergence of solution can be evaluated according to the criteria such as online performance and 
offline performance, while the GAs' performance is measured by the value of objective function through the best chromosome (best solution criterion).The GAs' peculiarities are summarised in the following:

- they don't be influenced by the number of variables;

- they can face non linear problems;

- they search a solution as population;

- they use only objective function, neither derivate nor others;

- they follow probabilistic rules for transition.

The GAs are frequently used to solve optimisation multi-objective problems which are characterised by two or more objective functions.

\section{Background}

GAs have found wide application in the field of the road asset and infrastructure since 1994 [1].

Several issues were tackled by GAs because this approach was considering performing in order mainly to solve problems of resources allocation for road pavement maintenance and rehabilitation [2], [3], [4], [5], [6], [7], [8] but also to improve pavement design and analysis [9], [10], [11], [12], [13], [14], [15] and highway alignment design [16].

In particular, the research addressed to the pavement management system (PMS) was performed with continuity over the last two decades.

With regards the problem of finding multi-year pavement repair schedule Tack and Chou [2] compared GAs and a dynamic programming (DP) approach. In particular two types of GAs, simple and pre-constrained (PCGA) were used. The authors considered one objective function as overall average yearly network condition that depends on pavement conditions of each section selected. They also introduced the concept of the state of the system in terms of budget to be spent on repair technique for section " $\mathrm{i}$ " in year " $\mathrm{j}$ ". The results proved the substantial convergence of the three different procedures (DP, SGA, PCGA) even if the overall convergence time was less than that for SGA.

Cheu et al. [3] dealt with the influence of pavement maintenance activities on road users travel time, searching the scheduling of this activities that minimized such travel time. A GAs procedure coupled with the traffic simulation model (hybrid method) was used in order to optimize the maintenance activities plan, obtaining relevant results.

Instead Bosurgi and Trifirò [4] used an hybrid model based on GAs-ANN (Artificial Neural Network) to optimize the problem of the pavement maintenance. The optimization process was faced by opportunely programming GAs procedure according to two indicators: sideway force coefficient and predicted accident, both defined through models elaborated with ANN.

Chootinan et al. [5] dealt with a stochastic simulation-based GAs approach addressed to a multi-year pavement maintenance program. The GAs approach were used for handling the combinatorial nature of the network level pavement programming while a stochastic simulation was performed in order to take in 
account the uncertainty due to entity of traffic on the predicted pavement deterioration. Regarding this issue the Markov transition probability matrix (TPM) approach appears to be the only model explicitly developed to address the uncertainty because it forecasts the distribution of future pavement condition over the entire network. They considered two objective functions (maintenance cost and pavement performance) while the constraints were the available budget and minimum condition level respectively.

Wang et al. [6] considered two conflictive objectives that are the cost minimization and pavement performance maximization. Starting from NOS (network optimization system) that was developed and implemented by Arizona Department of Transportation since the 1980s, the authors integrated GAs techniques and Markov TPM to solve the NOS problem at network level. It was demonstrated that GAsbased NOS provided big advantages in terms of substantial savings.

Wei et al. [7] dealt with a multi-objective optimization problem for rehabilitation strategies on project level. They established two objective functions such as the action costs and the user benefits in terms of performance of pavement section. In order to identify the family of non-dominated solutions according to the Pareto optimality concept, the authors evaluated the fitness of each chromosome by Pilson method, being the chromosome a multi-year maintenance strategy over 15 years.

The work of Golroo and Tighe [8] was aimed at selecting an optimum GAs structure to be applied for developing a maintenance scheme, having the scope to carry out a sensitivity analysis on outcomes with respect to GAs structure; both single objective function and two objectives functions were employed.

\section{Method}

The proposed model PMS-GA is aimed at solving two-objectives optimization problem on the basis of the definition of two functions, the risk state function (RSF) representing the degree of safety offered by the road infrastructure, and the costs of rehabilitation treatment.

The model can be applied both on network level and project level.

Preliminarily it is necessary to perform the following:

- Creation of a functional and geometric database, containing the characteristics of the infrastructures in question, arranged in functional unit FU;

- Creation of a database of accident rates, requiring selection of the factors that concur to cause an accident and the formulation of a function that links these factors to the accident rate itself.

\subsection{Selection of indicators of the state}

The first phase consists of acquiring data regarding the geometry of the route, the surface characteristics of the pavement, weather and climate conditions, and traffic divided into heavy and light vehicles. 
Each FU that is geometrically characterised in this way is a distinct element, defined by the same conditions of the state of pavement and climatic conditions.

Generally a set of indicators should made up at least of annual average daily traffic (AADT), international roughness (IRI), sideway force coefficient (SFC) and an accident rate parameter. selected to represent each FU

Since it could be not easy to work with the numerical values of input indicators, we decided to subdivide the variation interval of each indicator into classes in order to refer to a single representative value for an entire class, and then create identification initials for that class.

The number of classes for each indicator depends on the need to describe in detail the data and on the importance assigned to certain characteristics in terms of determining accident occurrence; for some of these characteristics, the extra detail is due to the fact that maintenance or rehabilitation intervention is based directly on these characteristics.

\subsection{Definition of the risk state function}

The RSF is formalised on the assumption that the relation linking the number of accidents to the chosen indicators, is linear for each of the considered functional units.

The total number of risk states depends on all the possible combinations of the identified classes of state indicators.

Therefore, each FU is characterised by a particular risk state value RS.

\subsection{Rehabilitation treatments}

The types of intervention to be carried out on the road in question depend on the state of wear of the pavement, and since this varies so much, a whole range of intervention solutions needs to be available.

The most common rehabilitation treatments can be subdivided in minor intervention such as surface enrichment, slurry sealing, split-mastic asphalt, overlay, and in major intervention such as resurfacing, reconstruction and full depth repair

Among the possible solutions, we must also include solution 0 , that is, the choice not to apply any solution, which has a corresponding cost, which allows for cleaning and inspection expenses.

An operative strategy on the entire road network is obtained when for each FU, the type of solution and the time for intervention is established.

The available budget, $\mathrm{B}$, for rehabilitation over a period of time, is a given factor in the problem, and depends upon the management policy of the company. Such a factor is a strong conditioning element, since out of the whole range of possible strategies for the network in question, one must first exclude all the strategies whose costs exceed the budget. 


\subsection{Application of Markov's theory}

Once an S series of possible strategies for intervention on the network has been assumed, it is necessary to predict the RS for each individual FU at the end of the rehabilitation work planning period.

The probability model for the forecast is the stationary Markovian model, based on the assumption that the probability at $t+1$, that the variable $X$, in this case the Risk State, will assume the value $i_{t+1}$ depends exclusively on the value of the variable considered corresponding to the time $t$ immediately before, and not on the sequence of values that $\mathrm{X}$ assumes in times $\mathrm{t}-1, \mathrm{t}-2, \ldots, 1,0$.

Indicating the probability of transition (one step), with $p_{i j}$, that is the probability that at time $t+1$ the system will be in state $j$, having been in state $i$ at time $t$, all the conditions of transition of the system from one state to another due to the effect of the generic intervention can be summed up in the $\mathrm{k}^{\text {th }}$ Transition Matrix:

$$
\overline{\overline{P_{k}}}=\left(\begin{array}{cccc}
p_{1,1} & p_{1,2} & \cdots & p_{1, m} \\
p_{2,1} & p_{2,2} & \cdots & p_{2, m} \\
\cdots & \cdots & \cdots & \cdots \\
p_{m, 1} & p_{m, 2} & \cdots & p_{m, m}
\end{array}\right)
$$

$\overline{\overline{P_{k}}}$ is a square matrix of $\mathrm{m}^{*} \mathrm{~m}$ elements, for which the following condition is given:

$$
\sum_{j=1}^{m} p_{i j}=1
$$

The number of transition matrices is equal to the possible typologies of intervention. Each element of the transition matrix is defined as a composite transition and is the probability that the set of indicators placed respectively in classes a, b, c, d before the intervention, move to classes e, $\mathrm{f}, \mathrm{g}, \mathrm{h}$ as a consequence of intervention $\mathrm{k}$ in case of 4 indicators. Such probability is obtained as a product of each single simple transition probability of each indicator, considered as independent events.

Therefore if the combination of classes [a, b, c, d] represents the generic risk state $\mathrm{i}$, while the combination [e, f, g, h] represents the status $\mathrm{j}$, the transition $p_{i j}$ of the generic UF is computed as follows:

$$
p_{i j}=P(a, b, c, d \rightarrow e, f, g, h)=P_{I_{1} a e} \times P_{I_{2} b f} \times P_{I_{s} c g} \times P_{I_{d} d h}
$$

The single factor of the product is the transition probability of one of the 4 considered status indicators and can be computed analyzing the historical data tracing the percentages of networks that pass from one class to the other of the generic indicator during a year as a consequence of the $\mathrm{k}^{\text {th }}$ intervention [17].

The reference database concerning the analysis period provides the necessary information to determine the probability, as described above, on the basis of the chronology and typology of rehabilitation intervention performed. 
The variables of the optimization problem are the percentages of length of the road network $\Lambda_{r}^{a}$ which are in a given risk state $\mathrm{r}$, during the generic year $a$ of the programming period.

If we assume that year $a$ is the moment in which we plan the interventions for the following year $a+1$, the current state of the road pavement is that of year $a$, while the road pavement conditions expected as a consequence of the intervention strategy selected are those of the year $a+1$.

In addition we indicate as $\lambda_{r k}^{a}$ the length percentage of the entire road network that in the year $a$ is at state $\mathrm{r}^{\text {th }}$ and is programmed to undergo the generic intervention $\mathrm{k}$, and as $\lambda_{r k}^{a+1}$ the length percentage that in the year $a+1$ is at state $\mathrm{r}$ as a consequence of the $\mathrm{k}^{\text {th }}$ intervention.

The formulas are:

$$
\Lambda_{r}^{a}=\frac{L_{r}^{a}}{L_{t o t}} \quad \lambda_{r k}^{a}=\frac{L_{r k}^{a}}{L_{t o t}}
$$

Thus defined the variables of the problem, naturally we obtain:

$$
\Lambda_{r}^{a}=\sum_{k=0}^{q} \lambda_{r k}^{a} \quad \quad \sum_{k=0}^{q} \sum_{r=1}^{m} \lambda_{i k}^{a}=1 \quad \sum_{k=0}^{q} \sum_{r=1}^{m} \lambda_{i k}^{a+1}=1
$$

To define the initial conditions it is necessary to compute the length quantities of the road network which are in one of the m risk states during year $a$, the year in which planning takes place. We obtain a vector $\bar{\Lambda}^{a}$ of dimension $\mathrm{m}$ that is made of the $\mathrm{m}$ length percentages $\Lambda_{r}^{a}$.

Within quantity $\Lambda_{r}^{a}$ a component will undergo intervention 0 , one component intervention 1, and another component generic intervention $\mathrm{k}$ and so forth, therefore for each intervention strategy we obtain the k vectors $\bar{\lambda}_{k}^{a}$ of simple percentages $\lambda_{r k}^{a}$ :

$$
\bar{\Lambda}^{a}=\bar{\lambda}_{0}^{a}+\bar{\lambda}_{1}^{a}+\bar{\lambda}_{2}^{a}+\ldots+\bar{\lambda}_{q}^{a}
$$

which written as a matrix is:

$$
\bar{\Lambda}^{a}=\left[\begin{array}{c}
\Lambda_{1}^{a} \\
\Lambda_{2}^{a} \\
\ldots \\
\Lambda_{m}^{a}
\end{array}\right]=\left[\begin{array}{c}
\lambda_{1,0}^{a} \\
\lambda_{2,0}^{a} \\
\ldots \\
\lambda_{m, 0}^{a}
\end{array}\right]+\left[\begin{array}{c}
\lambda_{1,1}^{a} \\
\lambda_{2,1}^{a} \\
\ldots \\
\lambda_{m, 1}^{a}
\end{array}\right]+\left[\begin{array}{c}
\lambda_{1,2}^{a} \\
\lambda_{2,2}^{a} \\
\ldots \\
\lambda_{m, 2}^{a}
\end{array}\right]+\ldots+\left[\begin{array}{c}
\lambda_{1, q}^{a} \\
\lambda_{2, q}^{a} \\
\ldots \\
\lambda_{m, q}^{a}
\end{array}\right]
$$

The transition conditions are represented by the equation that expresses the product of the $\mathrm{r}^{\text {th }}$ column of the transition matrix of the generic intervention $\mathrm{k}$ for the vector $\bar{\lambda}_{k}^{a}$. 
Such scalar product results in a numerical value expressing the sum of the network percentages which as a consequence of the intervention $\mathrm{k}$ pass from the $\mathrm{m}$ risk states in which they were during year $a$ to the $\mathrm{j}^{\text {th }}$ risk state in year $a+1$.

$$
\begin{gathered}
\lambda_{j k}^{a+1}=\sum_{r=1}^{m}\left(\lambda_{r k}^{a} \cdot P_{k}(r, j)\right) \\
\Lambda_{j}^{a+1}=\sum_{k=0}^{4} \lambda_{j k}^{a+1}=\sum_{r=1}^{m} \sum_{k=0}^{q}\left(\lambda_{r k}^{a} \cdot P_{k}(r, j)\right)
\end{gathered}
$$

In matrix form the first of the two relations, specifically for intervention 0 , becomes:

$$
\left[\begin{array}{c}
\lambda_{1,0}^{a+1} \\
\lambda_{2,0}^{a+1} \\
\ldots \\
\lambda_{m, 0}^{a+1}
\end{array}\right]=\left[\begin{array}{cccc}
P_{0}(1,1) & P_{0}(2,1) & \ldots & P_{0}(m, 1) \\
P_{0}(1,2) & P_{0}(2,2) & \ldots & \ldots \\
\ldots & \ldots & \ldots & \ldots \\
P_{0}(1, m) & \ldots & \ldots & P_{0}(m, m)
\end{array}\right] *\left[\begin{array}{c}
\lambda_{1,0}^{a} \\
\lambda_{2,0}^{a} \\
\ldots \\
\lambda_{m, 0}^{a}
\end{array}\right]
$$

Note that the transition matrix of the matrix product is not the $\overline{\overline{P_{0}}}$ of the Equation (4), but its transposed.

The vector $\bar{\Lambda}^{a}$ represents the distribution of the network percentages during the various risk states before carrying out the generic maintenance strategy s, the vector $\bar{\Lambda}^{a+1}$ represents the same distribution as a consequence of the strategy s $\left(\bar{\Lambda}^{a} \neq \bar{\Lambda}^{a+1}\right)$.

The first goal function of the programming model is a measure of the danger level of the road network and represents the degree of safety and functionality of the infrastructure following the maintenance interventions selected for each basic section of the network under examination. This can be computed with the following formula:

$$
\Theta=\sum_{r=1}^{m} \sum_{k=0}^{q} \lambda_{r, k}^{a+1} \cdot R S_{r}
$$

The second goal function matches indeed the cost Cs of each intervention strategy, computed with the following formula:

$$
C_{S}=\sum_{m=1}^{M} \sum_{k=0}^{q} L_{m k}^{a} \cdot C_{k}
$$

where $\mathrm{L}_{m}$ is the longitudinal extension of the $\mathrm{m}^{\text {th }}$ Functional Unit $(\mathrm{m}=1,2, \ldots, \mathrm{M})$.

The two criteria are reasonably in contrast. In order to considerably reduce the danger level of the network it is necessary to intervene with more incisive therapies, therefore spending a bigger amount of resources. If a minor cost strategy is selected obviously you cannot solve the functionality conditions of the whole network, 
instead an intervention priority must be decided for those areas that present major criticality for the circulation safety.

\subsection{Optimisation GA procedure}

The calculation process of the Genetic Algorithm follows a set of elementary instructions that are applied repeatedly until obtaining convergence to a set of solutions known as the Pareto optimal front.

The original population of chromosomes, each representing a maintenance strategy, is generated randomly. The main steps of each application are:

1. Selection of a population of popSize elements using the Roulette Wheel selection procedure: once the cumulative fitness has been generated and a random number between zero and the fitness rank has been extracted from the whole population (as a sum of all the fitness), there is a selection of the individual with a fitness rank immediately below the extracted random number. This procedure is applied as many times as there are individuals in the population.

2. Updating of the population applying crossover and mutation operators.

The first is applied after random popSize/2 selection of couples of individuals. Then the mutation operator randomly selects a gene from each string, and with a probability that depends on the rate of mutation, substitutes the value with one of five possible values $(0,1, \ldots, 4)$.

3. Calculation of the $\mathrm{W}$ matrices of the percentages of road pavement that are found respectively in the various risk states and that are to undergo different maintenance interventions according to the proposed strategies.

Matrix products are calculated to obtain the WW matrices of the same percentages expected following maintenance, and finally, risk state functions and costs for each population string are calculated.

4. Classification of the solutions according to the following dominance rules:

$f_{1}\left(x_{1}\right)<f_{1}\left(x_{2}\right)$ e $f_{2}\left(x_{1}\right) \leq f_{2}\left(x_{2}\right)$ or $f_{1}\left(x_{1}\right) \leq f_{1}\left(x_{2}\right)$ e $f_{2}\left(x_{1}\right)<f_{2}\left(x_{2}\right)$

Dummy Fitness values are assigned which are the inverse of the rank, substituting previously obtained fitness ranks with this single value which represents a compromise of the two.

5. Adoption of fitness cut techniques using the niche method to avoid premature convergence of the genetic algorithm.

6. Calculation of the cumulative fitness using values obtained after the cut technique.

The process is repeated the same number of times as the number of generations, placing the population fitness values in a matrix entitled 'Genetic Algorithm Results'.

This matrix includes all the solutions provided by the Algorithm throughout the various generations, from which the best or dominant solutions are selected, representing the "Pareto Front".

In order to select the Optimum Final Strategy (OFS) from this curve, various criteria such as minimum cost $\left(\min \left[\mathrm{C}_{\mathrm{i}}\right]\right)$, minimum accident rate $\left(\left[\min \left[\Theta_{\mathrm{i}}\right]\right)\right.$, minimum marginal cost $\left(\min \left[\mathrm{MC}_{\mathrm{i}}\right]\right)$ and minimum distance $\left(\min \left[\mathrm{Dist}_{\mathrm{i}}\right]\right)$ may be followed. 
The minimum marginal cost expressed as:

$$
M C_{i}=\frac{C_{i}}{\Theta_{\text {initial }}-\Theta_{i}}\left[€ /\left(\mathrm{n}^{\circ} \text { accidents } \mathrm{x} \mathrm{FU}\right)\right]
$$

where $\Theta_{\text {initial }}$ is the danger in year $a$ before carrying out any intervention, and is obtained as follows:

$$
\Theta_{\text {initial }}=\sum_{r=1}^{R} \sum_{k=0}^{K} \lambda_{r, k}^{a} \cdot R S_{r}
$$

The minimum distance expressed as:

$$
\text { Dist }_{\mathrm{i}}=\sqrt{\left(\frac{\Theta_{i}-\Theta_{\min }}{\Theta_{\max }-\Theta_{\min }}\right)^{2}+\left(\frac{C_{i}-C_{\min }}{C_{\max }-C_{\min }}\right)^{2}}
$$

\section{Case study}

The procedure described above was applied to the A18 (Messina-Catania) motorway with the aim to optimise the rehabilitation activities over the pavement of the entire route considering a "year-by-year" horizon planning.

The accident risk in terms of RSF and rehabilitation costs C were analyzed as objective functions to be minimized.

The A18 was subdivided in $114 \mathrm{FU}$, defined by the same conditions of the state of pavement and climatic conditions. By the light of previous studies carried out on the A18 [18] through the use of ANN had already identified, using a precise hierarchical scale, the pre-existing causes that determine accidents.

In this case only the causes varying over time, called risk state indicators (RSI), were considered; in other words the alignment parameters such as curvature change rate (CCRs), grade and other geometric features were not included.

The RSI coming from the agency database were AADT, IRI, SFC and a climate index $I_{k}$ expressed as ratio between the annual rainfall height and the rainy days considering the mean values over the last three decades.

The period of analysis covers eight years from 2000 to 2007; since it is not easy to work with the numerical values of input RSI, we decided to subdivide the variation interval of each indicator into classes in order to refer to a single representative value for an entire class, and then create identification initials for that class.

The number of classes for each indicator depends on the need to describe in detail the data and on the importance assigned to certain characteristics in terms of determining accident occurrence; for some of these characteristics, the extra detail is due to the fact that rehabilitation intervention is based directly on these characteristics.

The following table 1 shows the variation domain and the classes for each indicator. 


\begin{tabular}{|c|c|c|}
\hline Indicator & Ranges of domain & N. classes \\
\hline IRI & $1-4.15$ & 3 \\
\hline SFC & $40.5-70.5$ & 3 \\
\hline AADT & $2800-26800$ & 3 \\
\hline Ik & $1.0-7.0$ & 2 \\
\hline Accident range & $0-17$ & \\
\hline
\end{tabular}

Table 1: Variation domain ranges.

Besides, another indicator $\mathrm{Y}$ representing the time was considered since an impressive pavement rehabilitation intervention was occurred around 2004 over nearly entire the motorway.

The non-linear regression Poisson's model with random effect using a panel database was performed [19] on a sample of about 380 observations.

The implementation of the model was achieved using STATA; after several tests, the $\mathrm{I}_{\mathrm{k}}$ parameter was resulted not relevant and the following relation was obtained on a final sample of 368 observations according to Hausman's test:

$$
S R=e^{0.8874524-0.0298444 \cdot C A T+0.2912722 \cdot I R I+0.0000249 \cdot T G M+0.0855481 \cdot Y}
$$

The rehabilitation treatments considered and their cost are summarized in table 2 according to a Sicilian market survey regarding the most common major rehabilitation solutions for motorway pavement. Among the possible solutions, we must also include solution 0 , that is, the choice not to apply any solution, which has a corresponding cost of 13 Euros which allows for cleaning and inspection expenses.

\begin{tabular}{|c|c|}
\hline Rehabilitation Intervention & Cost [€/m] \\
\hline No Treatment (0) & 13.00 \\
\hline Surface Repair (1) & 90.00 \\
\hline Coating Repair (2) & 210.00 \\
\hline Asphalt layers Reconstruction (3) & 380.00 \\
\hline Full Depth Repair (4) & 595.00 \\
\hline
\end{tabular}

Table 2: Treatments and cost.

An example of rehabilitation strategy year-by year as chromosome with 114 genes is represented in Figure 1. Each cell expresses a rehabilitation treatment which should be implemented on a FU. 


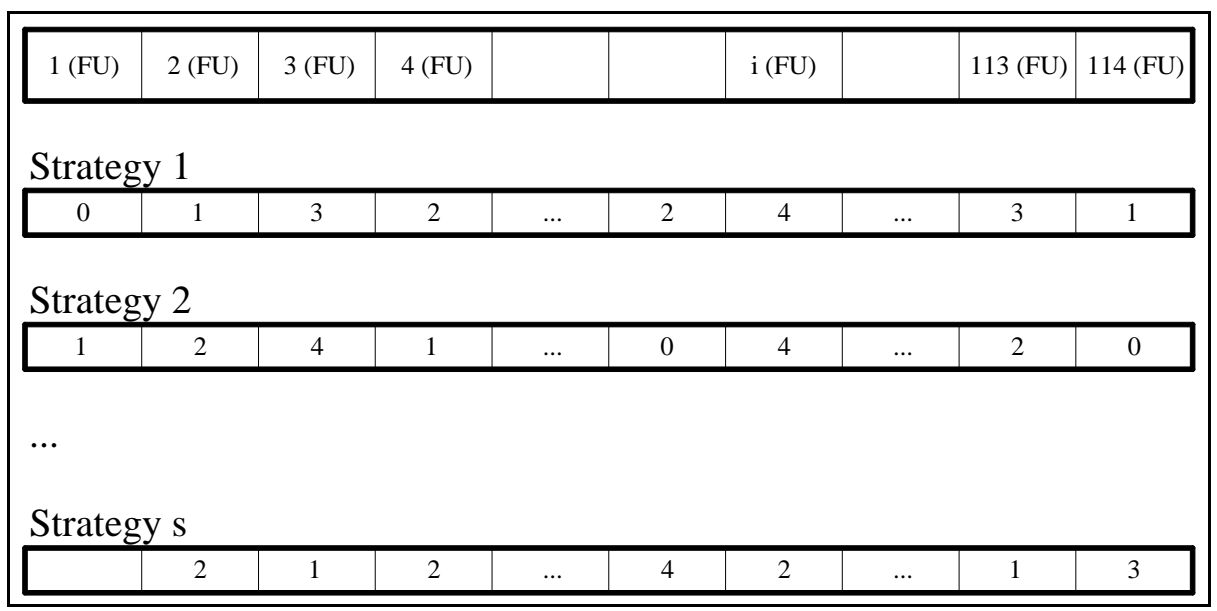

Figure 1: Structure of the chromosome.

The implementation of the GA procedure was achieved using Wolfram MATHEMATICA 7.0. The model was applied by acting on the generation of chromosome populations: for instance in the starting population, made up of 100 chromosomes, 5 extreme chromosomes were inserted, these represented a strategy consisting in the same intervention on the whole route, while 95 was entirely generated randomly. Every starting population (POPsize $=100 ; 150 ; 200 ; 500)$ was exposed to an evolutionary process according to 100, 150, 200 and 500 iterations, varying the mutation rate $(\mathrm{MR}=0.8 ; 0.9)$ and the ray of niche $\left(\sigma_{\mathrm{Sh}}=0.7 ; 0.9\right)$ with a crossover rate equal to 0.8 and roulette wheel as selection procedure. According to the adopted approach not completely random, the number of optimal solutions (Figure 2) and convergence (Figure 3) provided good results both from a quantitative (number of distinct optimal solutions) and qualitative (chosen solution according to the criterion of the minimum distance) point of view [20].

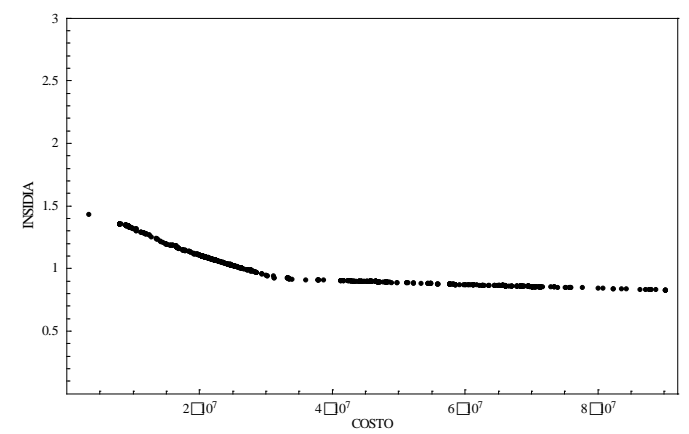

Figure 2: Pareto's Front and “near optimal solution” (Popsize100, Gen100, MR $\left.0.8 \sigma_{\text {sh }} 0.7\right)$.

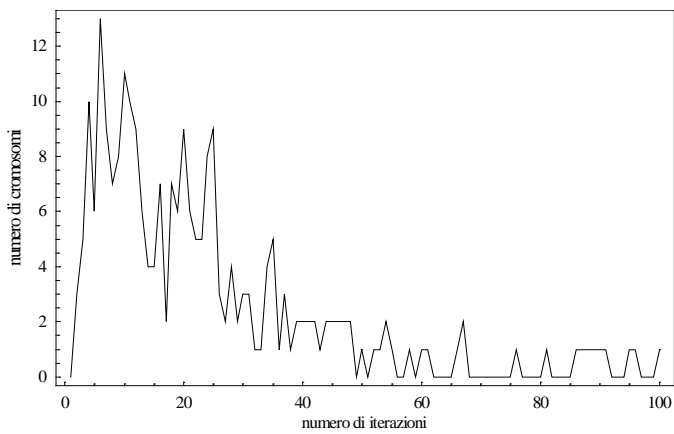

Figure 3: Convergence process of "near optimal solution" (Popsize100, Gen100-MR $\left.0.8 \sigma_{\mathrm{sh}} 0.7\right)$. 


\section{Results and discussion}

By means of a sensitivity analysis carried out over all combinations no univocal trend of outcomes such as chromosomes on Pareto's front (CPF) was identified (Table 3). It confirmed the heuristic nature of the GA approach.

\begin{tabular}{|c|c|c|c|c|c|c|c|}
\hline \multicolumn{2}{|c|}{ Gen. 100 - MR $0.8-\sigma_{\text {sh }} 0.7$} & \multicolumn{2}{|c|}{ Gen. 100 - MR 0.9 - $\sigma_{\text {sh }} 0.7$} & \multicolumn{2}{|c|}{ Gen. 100 - MR 0.8 - $\sigma_{\text {sh }} 0.9$} & \multicolumn{2}{|c|}{ Gen. 100 - MR 0.9 - $\sigma_{\text {sh }} 0.9$} \\
\hline POP & CPF & POP & CPF & POP & CPF & POP & CPF \\
\hline 100 & 171 & 100 & 304 & 100 & 129 & 100 & 269 \\
\hline 150 & 243 & 150 & 359 & 150 & 415 & 150 & 277 \\
\hline 200 & 280 & 200 & 301 & 200 & 244 & 200 & 169 \\
\hline 500 & 173 & 500 & 244 & 500 & 41 & 500 & 16 \\
\hline \multicolumn{2}{|c|}{ Gen. 150 - MR 0.8 - $\sigma_{\text {sh }} 0.7$} & \multicolumn{2}{|c|}{ Gen. 150 - MR 0.9 - $\sigma_{\text {sh }} 0.7$} & \multicolumn{2}{|c|}{ Gen. 150 - MR 0.8 - $\sigma_{\mathrm{sh}} 0.9$} & \multicolumn{2}{|c|}{ Gen. 150 - MR 0.9 - $\sigma_{\text {sh }} 0.9$} \\
\hline POP & CPF & POP & CPF & POP & CPF & POP & CPF \\
\hline 100 & 361 & 100 & 298 & 100 & 240 & 100 & 247 \\
\hline 150 & 420 & 150 & 260 & 150 & 291 & 150 & 283 \\
\hline 200 & 288 & 200 & 129 & 200 & 134 & 200 & 475 \\
\hline 500 & 140 & 500 & 49 & 500 & 84 & 500 & 79 \\
\hline \multicolumn{2}{|c|}{ Gen. 200 - MR 0.8 - $\sigma_{\text {sh }} 0.7$} & \multicolumn{2}{|c|}{ Gen. 200 - MR 0.9 - $\sigma_{\text {sh }} 0.7$} & \multicolumn{2}{|c|}{ Gen. 200 - MR 0.8 - $\sigma_{\text {sh }} 0.9$} & \multicolumn{2}{|c|}{ Gen. 200 - MR 0.9 - $\sigma_{\text {sh }} 0.9$} \\
\hline POP & $C P F$ & POP & CPF & POP & CPF & POP & CPF \\
\hline 100 & 215 & 100 & 599 & 100 & 657 & 100 & 221 \\
\hline 150 & 488 & 150 & 129 & 150 & 601 & 150 & 273 \\
\hline 200 & 244 & 200 & 86 & 200 & 299 & 200 & 571 \\
\hline 500 & 204 & 500 & 229 & 500 & 216 & 500 & 439 \\
\hline \multicolumn{2}{|c|}{ Gen. 500 - MR 0.8 - $\sigma_{\text {sh }} 0.7$} & \multicolumn{2}{|c|}{ Gen. 500 - MR 0.9 - $\sigma_{\text {sh }} 0.7$} & \multicolumn{2}{|c|}{ Gen. 500 - MR 0.8 - $\sigma_{\mathrm{sh}} 0.9$} & \multicolumn{2}{|c|}{ Gen. 500 - MR 0.9 - $\sigma_{\text {sh }} 0.9$} \\
\hline POP & $C P F$ & POP & CPF & POP & CPF & POP & $C P F$ \\
\hline 100 & 417 & 100 & 779 & 100 & 657 & 100 & 110 \\
\hline 150 & 683 & 150 & 421 & 150 & 662 & 150 & 644 \\
\hline 200 & 417 & 200 & 462 & 200 & 685 & 200 & 698 \\
\hline 500 & 524 & 500 & 432 & 500 & 701 & 500 & 653 \\
\hline
\end{tabular}

Table 3: Overview of number of best solutions.

According to every criterion mentioned in 4.3., the best chromosome (rehabilitation strategy) was identified due to maximum number of recurrence over the optimal solution set for all considered combinations equal to 64 .

As it can be expected, we obtained the chromosome "ALL0" both for $\min \left[\mathrm{C}_{\mathrm{i}}\right]$ and $\min \left[\mathrm{MC}_{\mathrm{i}}\right]$ and the chromosome "ALL4" for $\min \left[\Theta_{\mathrm{i}}\right]$ criterion respectively (Figure 4). 


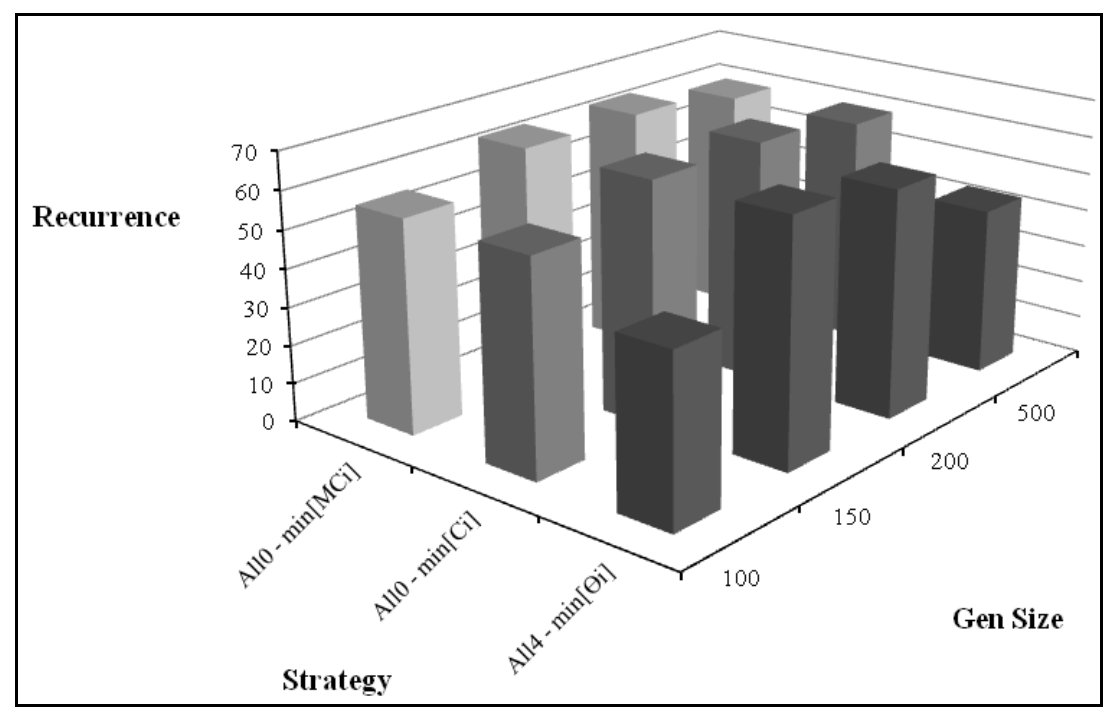

Figure 4: Recurrence of best solution for three criterion.

Therefore the $\min \left[\mathrm{Dist}_{\mathrm{i}}\right]$ criterion seems to be more suitable to solve the optimization problem. Conceptually speaking such criterion summarizes the search of arrangement solution. Every selected chromosome over all 64 combinations together with the extreme solutions as "ALL0" and "ALL4" (red points) are reported on the chart below (Figure 5). According to this criterion the best strategy (green point) costs about 15 millions Euros.

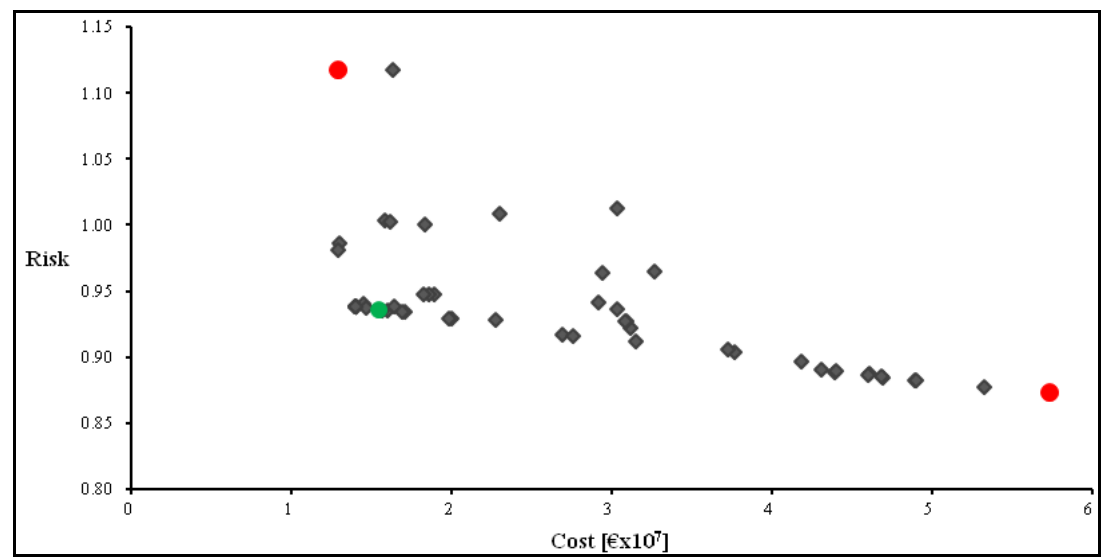

Figure 5: "Near optimal solution” according to minimum distance criterion.

This amount turned out to be lower than those of $70 \%$ other strategy considered.

Since the budget for pavement maintenance and rehabilitation are established, over either multi-year or year by year planning horizon, the proposed GA model allows to implement an effective Pavement Management System because on the basis of the required objective such as the road safety, also the expected performance is established. 


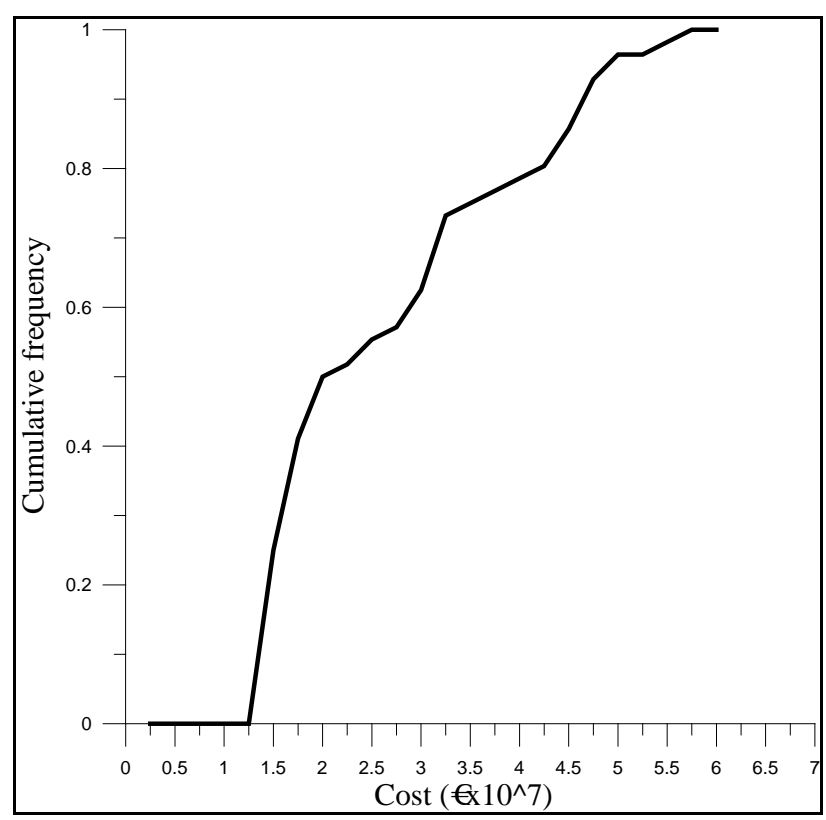

Figure 6: Frequency distribution of rehabilitation strategy cost.

\section{Summary and conclusion}

A GA based procedure was developed to solve a two-objectives optimization problem taking in account road accident risk and rehabilitation costs as objective functions to be minimised, both on network level and project one.

The GA based procedure was performed on real case concerning the A18 MessinaCatania motorway in Italy and the results demonstrated that the GAs procedure was able to reach technical and economical targets using a specific criterion on the whose basis the best strategy was identified, at least in heuristic terms.

In future, further study, typically required for research of this kind, should focus on the formulation of a user's cost function as third objective function to be minimised so that these results, already important in themselves, may become even more so.

\section{References}

[1] W.T. Chan, T.F. Fwa and C.Y. Tan, "Road-maintenance planning using genetic algorithms, journal of Transport Engineering, 120, 693-709, 1994.

[2] J.N. Tack, E.Y.J. Chou, "Multiyear pavement repair scheduling optimization by preconstrained genetic algorithm”, Transportation Research Record, 1816, 3-9, 2002.

[3] R.L. Cheu, Y. Wang amd T.F. Fwa, “Genetic Algorithm-Simulation Methodology for Pavement Maintenance Scheduling, Computer-Aided Civil and Infrastructure Engineering 19, 446-455, 2004. 
[4] G. Bosurgi, F. Trifirò, "A model based on artificial neural networks and genetic algorithm for pavement maintenance management", International Journal of Pavement Engineering, 6(3), 201-209, 2005.

[5] P. Chootinan, A. Chen, M.R. Horrocks and D. Bolling, "A multi-year pavement maintenance program using a stochastic simulation-based genetic algorithm approach”, Transportation Research Part A, 40, 725-743, 2006.

[6] K.C.P. Wang, V. Nguyen and J.P. Zaniewski, "Genetic Algorithms-Based Network Optimization System with Multiple Objectives", Transportation Research Record, 2016, 85-96, 2007.

[7] J.J. Wei, Y.J. Kong and Z. Fu, " Multi-objective optimization for pavement maintenance and rehabilitation strategies", Proceedings of the 2nd International Conference on Transportation Engineering - ICTE, 345, 29192924, 2009.

[8] A. Golroo, S.L. Tighe, "Optimum Genetic Algorithm Structure Selection in Pavement Management”, Asian Journal of Applied Sciences, 5 (6), 327-341, 2012.

[9] A.R. Shekharan, " Solution of Pavement Deterioration Equations by Genetic Algorithms”, Transportation Research Record, 1699, 101-106, 2000.

[10] B.W. Tsai, V.N. Kannekanti and J.T. Harvey, "Application of Genetic Algorithm in Asphalt Pavement Design”, Transportation Research Record, 1891, 112-120, 2004.

[11] A. Pryke, H. Evdorides and R.A. Ermaileh, "Optimization of pavement design using a genetic algorithm", Proceedings of Congress on Evolutionary Computation - CEC, 1688431, 1095-1098, 2006.

[12] B.W. Tsai, J.T. Harvey and C.L. Monismith, " Case Studies of Asphalt Pavement Analysis/Design with Application of the Genetic Algorithm", Intelligence \& Soft Computing in Infrastructure System Engineering, 259, 205-238, 2009.

[13] S.W. Park, H.M. Park and J.J. Hwang, "Application of Genetic Algorithm and Finite Element Method for Backcalculating Layer Moduli of Flexible Pavements”, Journal of Civil Engineering, 14 (2), 183-190, 2010.

[14] W. Qian, "Road Pavement Performance Evaluation Model Based on Hybrid Genetic Algorithm Neural Network", Proceedings of the Second International Conference on Computational Intelligence and Natural Computing - CINC, 5643855, 209-212, 2010.

[15] E. Salari and X. Yu, "Pavement Distress Detection and Classification Using a Genetic Algorithm", Proceedings of Applied Imagery Pattern Recognition Workshop, 6176378, 2011.

[16] M.K. Jha, "Optimizing Highway Networks: A Genetic Algorithms and Swarm Intelligence Based Approach, Computing in Civil Engineering, 76-89, 2002.

[17] A Marchionna.- "L'innovazione nei materiali e nelle tecniche di manutenzione stradale." - Quaderni AIPCR - XXIV Convegno Nazionale Stradale, Saint Vincent - Aosta, 26-29 June. 2002

[18] A. Bevilacqua, G. Di Mino., M.Giunta.- "The analysis and diagnosis of car accidents on Sicilian freeways by Neural Network.” - Le Strade, Supplemento 
al n 1349, XXI World Road Congress Kuala Lumpur - Malaysia - October 39, special issue, pp. 224-229. 1999

[19] G. Di Mino., J. Nigrelli., A.Cirà.- “Analisi Before and After con modellazione multivariata” - Strade \& Autostrade, n 1349, pp. 224-229. 2009

[20] G. Di Mino., C.M. Di Liberto., F. Di Noto., J. Nigrelli., .- “ A multi objective approach based on the genetic algorithm for road pavement maintenance" Proceedings of the 4th International SIIV Congress - Palermo 12-14 September. 2007 